\title{
A MORE COMPREHENSIVE COMPLEMENTARY THEOREM FOR THE ANALYSIS OF POISSON POINT PROCESSES
}

\author{
RICHARD COWAN, ${ }^{*}$ University of Sydney
}

\begin{abstract}
In this paper we discuss the complementary theorem applied to the typical $n$-tuple of a Poisson point process. The theorem was first presented by Miles in 1970 and discussed by Santaló in 1976 and, within a Palm measure framework, by Møller and Zuyev in 1996. The theorems put forward by these authors are not correct for all the examples that they present, suggesting that further consideration of their work is needed if one wishes to bring all those examples within the ambit of the complementary theorem. We give alternative analyses of the errant examples and, with a modification of the technicalities in the work of the above authors, move toward a more comprehensive complementary theorem. Some open issues still remain.
\end{abstract}

Keywords: Random geometry; Poisson point process; Poisson flat process; complementary theorem; gamma distribution

2000 Mathematics Subject Classification: Primary 60D05; 60G55

\section{Introduction}

Consider firstly a Poisson point process in $\mathbb{R}^{2}$ of intensity $\rho$. This is also called a Poisson particle process by some authors, to allow easy distinction between the random 'particles' of the process and other points of the space.

Miles [5] showed that a random domain $\Delta$ constructed from a typical $n$-tuple of Poisson particles and containing $m \geq 0$ other particles (or ' $m$-filled' in Miles' terminology) has $\Gamma(n+$ $m-1, \rho)$-distributed area. He required some regularity conditions (which we discuss below in Section 2) on the way that $\Delta$ is created, but these conditions still allow a vast repertoire of imaginative and intricate constructions. Consider Figure 1(a), which shows a 5-filled realisation of a domain $\Delta$ constructed as follows.

Example 1. Define the diametrical disk of two particles $x_{1}, x_{2} \in \mathbb{R}^{2}$ as the disk having the line-segment $x_{1} x_{2}$ as a diameter. Let $n=3$. Draw all three diametrical disks based on the three possible pairs of particles and construct $\Delta$ as the union of the two largest disks minus the smallest disk.

Miles' powerful result tells us that the area of such a 5-filled $\Delta$ has a $\Gamma(7, \rho)$ distribution, if the selected 3-tuple of particles on which it is based is 'typical'. A direct verification of this result using not Miles' theorem but, rather, routine methods of integral calculus and geometry would be very complicated indeed.

Miles called his result the complementary theorem because the problem of finding the distribution of the area of a random domain filled with a given number of particles is the

Received 13 December 2004; revision received 23 May 2006.

* Postal address: School of Mathematics and Statistics, University of Sydney, NSW 2006, Australia.

Email address: rcowan@mail.usyd.edu.au 


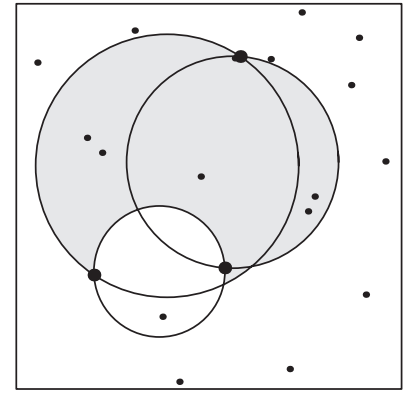

(a)

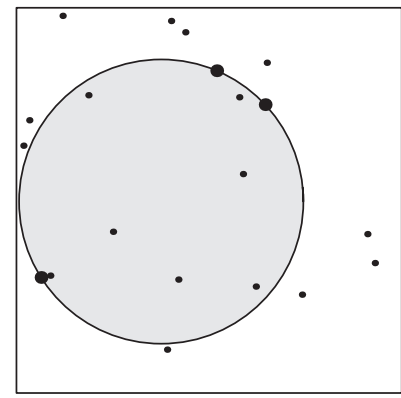

(b)

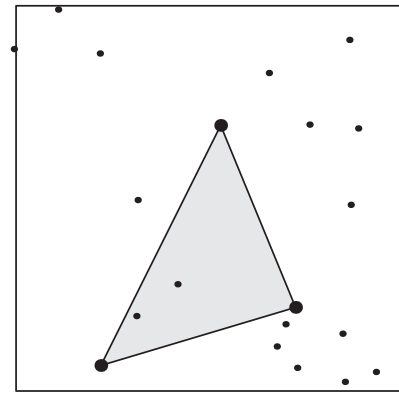

(c)

FIGURE 1: Three examples for $\Delta$ based on typical 3-tuples. In each, $\Delta$ is the shaded domain. (a) A typical $\Delta$ for Example 1. It happens to be 5-filled. (b) A 7-filled $\Delta$ based on Example 2, below. (c) A 2-filled $\Delta$ based on Example 3, below. The realised Poisson process extends beyond the observation window in each figure.

complement of a more direct problem, namely finding the distribution of the random count of particles within a given domain. In his later work [6], Miles generalised the theorem to deal with the volumes of domains constructed from Poisson particle processes in $\mathbb{R}^{d}$ - with further generalisation to constructions based on Poisson flat processes. His definition of a typical collection of $n$ particles (or flats) was an ergodic one.

In Theorem 4 of Møller and Zuyev [8], the result of Miles for $s$-dimensional flats within $d$-dimensional space is given, proved by those authors using a Palm measure definition of typicality. When $s=0$, that is, when the flats are points (or, as we say, particles), the result is a domain with $\Gamma(n+m-1, \rho)$-distributed volume - in agreement with Miles.

One difference between the authors is in the constructive use of information given in the $n$-tuple. Miles allowed $\Delta$ to depend on the order of the particles within the typical $n$-tuple; Møller and Zuyev did not, but their approach can be adjusted easily to allow this feature and we do this below when establishing our technical framework.

Another difference is the provision in [5] for domains $\Delta$ which could have zero volume for some $n$-tuples; the mathematics in [8] makes no allowance for this feature. Care on this issue is only important when $m=0$; Miles is careful in such cases to state the gamma-distributional results for volume $V$ as being conditional upon $V>0$ (thus avoiding the obvious mass of probability that would accrue at $V=0$ due to domains $\Delta$ of zero volume being 0 -filled almost certainly). The spirit of his complementary theorem requires a focus on domains of positive volume, which have a positive chance of being hit by other particles. Our treatment will allow this provision of Miles.

Two examples worked through in both [5] and [8], and again in [9, pp. 18-19], are for $n=3$ and $d=2$. One of these is treated incorrectly in all three studies.

Example 2. Define $\Delta$ as the closed circumdisk having the three particles on its boundary. See Figure 1(b).

Example 3. Define $\Delta$ as the convex hull of the three particles or, in other words, the triangle having the particles as vertices. See Figure 1(c).

In both these examples, the theorem says that an $m$-filled $\Delta$ has an area which is $\Gamma(m+2, \rho)$ distributed. We show below that this claim is unsustainable in the second problem, Example 3. 
The area derived in our analysis, admittedly one based on a new theory which is not yet complete in all aspects, is actually $\Gamma(m+1, \rho)$-distributed.

We have found other examples where the shape parameter of the gamma distribution is not what one would expect from a cursory application of the existing complementary theorems of [5] and [8]. These examples are presented in the course of our discussion.

The classical complementary theorem for typical $n$-tuples was discussed in [1], but none of the examples considered there violate the classical theorem of Miles, and so do not concern us here. Some different complementary theorems, for domains which are not constructed from typical $n$-tuples, have been presented: for domains which evolve as stopping sets, by Zuyev [12]; and, for domains uniquely determined by the realised Poisson process, in the early sections of [8]. The current paper does not discuss these.

We now proceed to develop our technical framework. In doing so, we define the main regularity condition of Miles (called the equivariance condition). We also introduce other conditions implicitly used by Miles and by Møller and Zuyev, being necessary for their and our results.

In due course we re-establish the 'classical' theorem, but with more clearly stated conditions; see Theorem 1 . These conditions (perhaps not initially meaningful to the reader in this early statement of Theorem 1) will be clarified by definitions and examples in Sections 2-6 (and by the proof in Section 6).

Theorem 1. (The classical complementary theorem revisited.) Consider a Poisson particle process in $\mathbb{R}^{d}$. For a typical $n$-tuple whose associated domain $\Delta$ has positive volume and is $m$-filled, the volume, $V$, of $\Delta$ is $\Gamma(n+m-1, \rho)$-distributed provided that

- the transformation, $\Theta$, which defines the construction of $\Delta$ is equivariant, nonrandom, and with no superfluous arguments;

- the resulting domain $\Delta$ has finite volume with probability 1; and

- $\lambda$, the intensity of the point process formed by anchors of all qualifying $n$-tuples, is finite and positive.

In Sections 7-12, further new results and discussions give insights into Theorem 1 and offer suggestions for extending the domain of applicability of the 'complementary theorem techniques' - especially to the case where $\lambda$ is not finite.

\section{Technical framework for the theorem}

Our notation follows the text of [11] in most respects, with some changes as our needs dictate. We are also conscious of compatibility with the logical flow of [5] and [8] (especially the latter, in this early stage of our discussion).

Let $(\Omega, \mathcal{A}, \mathrm{P})$ be the probability field and let $\left(\mathbb{R}^{d}, \mathscr{B}^{d}, v_{d}\right)$ be the usual measure space for $d$-dimensional space, $v_{d}$ being the Lebesgue measure on the Borel sets $\mathscr{B}^{d}$. Let $\mathbb{N}$ be the set of all $\sigma$-finite measures on $\mathscr{B}^{d}$ of the form $\varphi=\sum_{i} n_{i} \delta_{x_{i}}$, where the points $x_{i} \in \mathbb{R}^{d}$ are distinct and the integers $n_{i} \geq 1$ are the point multiplicities. Here $\delta_{x}$ is the Dirac measure on $\mathscr{B}^{d}$ and, so, each $\varphi \in \mathbb{N}$ is a counting measure. We endow $\mathbb{N}$ with a $\sigma$-field $\mathcal{N}$ which is generated by all sets of the form $\{\varphi \in \mathbb{N}: \varphi(B)=n\}$ for $B \in \mathscr{B}^{d}$ and $n=0,1,2, \ldots$

Let $\Phi$ be the stationary Poisson particle process on $\mathbb{R}^{d}$ with intensity $\rho>0$. In other words, $\Phi$ is a mapping from $\Omega$ to $\mathbb{N}$ having the defining characteristic of a stationary Poisson particle process, namely that, for disjoint sets $B_{1}, \ldots, B_{k} \in \mathcal{B}^{d}$, the counts $\Phi\left(B_{1}\right), \ldots, \Phi\left(B_{k}\right)$ 


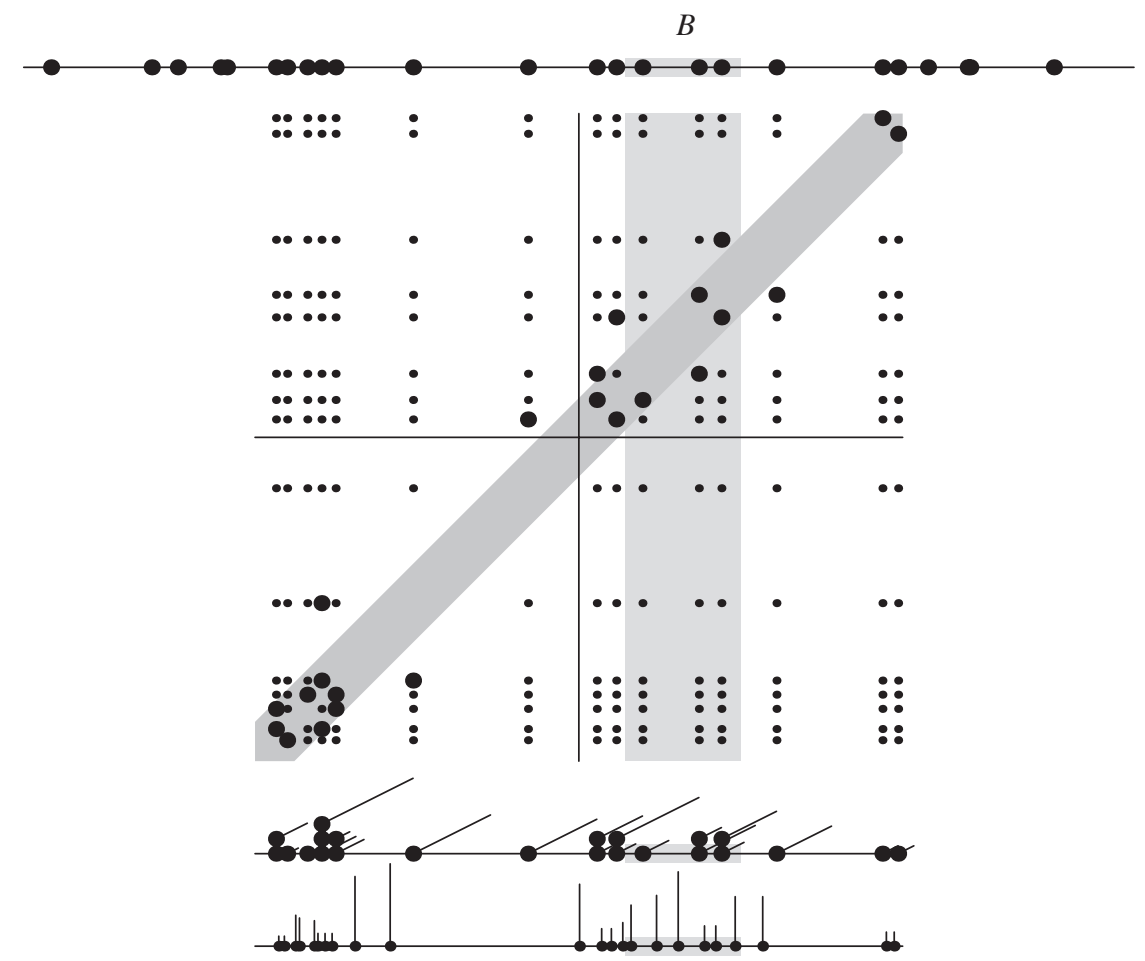

Figure 2: At the top is the realisation of a Poisson particle process on $\mathbb{R}$ shown within $I:=[-L, L]$. Below is the product process on $J \times J \subset \mathbb{R}^{2}$, where $J \subset I$. Heavier dots in the product process indicate that the associated domain $\Delta$, using the example mapping in Example 4, below, is 0 -filled. At the bottom are two marked point processes constructed from the product process in a manner discussed in the text. The marks are given by the lengths of lines extending upward from the points. Note that germs in the darkly shaded region have domains $\Delta$ with volume (length) less than $v$.

are mutually independent random variables. These counts are also Poisson distributed, $\Phi\left(B_{i}\right)$ having mean $\rho v_{d}\left(B_{i}\right)$. The particles $x_{i}$ each have multiplicity $n_{i}=1$ in the process $\Phi$.

Since we have an interest in $n$-tuples of distinct particles of $\Phi$, we consider an $n$-fold product process: a Cartesian product of $\Phi$, modified to remove $n$-tuples which have two or more equal components. For example, when $n=2$ we define

$$
\Phi^{[2]}(A \times B):=\Phi(A) \Phi(B)-\Phi(A \cap B)
$$

for $A, B \in \mathscr{B}^{d}$ and extend this to $\left(\mathscr{B}^{d}\right)^{2}$, all Borel sets of $\mathbb{R}^{d} \times \mathbb{R}^{d}$. The random counting measure $\Phi^{[2]}$ is a particle process (not Poisson) on $\mathbb{R}^{d} \times \mathbb{R}^{d}$ having intensity $\rho^{2}$. Figure 2 shows the 'product process' for an example in which $d=1$ and $n=2$.

For a general $n$, we can use the following construct as our definition of $\Phi^{[n]}$ on $\left(\mathcal{B}^{d}\right)^{n}$, creating a random 'product process' on $\left(\mathbb{R}^{d}\right)^{n}$ with intensity $\rho^{n}$ :

$$
\Phi^{[n]}\left(\mathrm{d} x_{1} \times \cdots \times \mathrm{d} x_{n}\right):=\Phi\left(\mathrm{d} x_{1}\right)\left(\Phi-\delta_{x_{1}}\right)\left(\mathrm{d} x_{2}\right) \cdots\left(\Phi-\sum_{i=1}^{n-1} \delta_{x_{i}}\right)\left(\mathrm{d} x_{n}\right) .
$$

For ease of reference, we call these particles in the product process germs. 
For each germ in the product process or, equivalently, for each $n$-tuple of original Poisson particles, we wish to define a domain $\Delta \in \mathscr{B}^{d}$ of finite volume. We do this by defining a mapping $\Theta$ from $\left(\mathbb{R}^{d}\right)^{n}$ to $\mathscr{B}^{d}$ such that the resulting domain, $\Delta:=\Theta\left(x_{1}, x_{2}, \ldots, x_{n}\right)$, has finite volume for all $\left(x_{1}, x_{2}, \ldots, x_{n}\right) \in\left(\mathbb{R}^{d}\right)^{n}$. We also impose the condition that $\Theta$ be nonrandom, have no superfluous arguments (that is, any $\Theta$ defined by a proper subset of the components of the $n$-tuple is not allowed), and have a form of 'scale and translation equivariance' as follows.

Let $\boldsymbol{a}$ be a transformation of the space $\mathbb{R}^{d}$ of the form $\boldsymbol{a} x=\alpha x+y$ for all $x \in \mathbb{R}^{d}$, where $\alpha>0$ and $y \in \mathbb{R}^{d}$. The set of all such transformations is denoted by $\mathcal{T}$. Note that $\mathcal{T}$ is a subset of the set of affine transformations. We require that $\Theta$ satisfy

$$
\Theta\left(\boldsymbol{a} x_{1}, \boldsymbol{a} x_{2}, \ldots, \boldsymbol{a} x_{n}\right)=\boldsymbol{a} \Theta\left(x_{1}, x_{2}, \ldots, x_{n}\right)
$$

for all $\boldsymbol{a} \in \mathcal{T}$. Miles, who imposed this condition, referred to such $\Theta$ as homothetically invariant but we shall use the term equivariant. Each germ is associated to its domain $\Delta$ via the equivariant mapping $\Theta$.

We note in passing that this form of equivariance implies that $n \geq 2$ (except in the trivial $n=1$ case, where $\Theta\left(x_{1}\right)=\left\{x_{1}\right\}$, a one-point set) and that the domain $\Delta$ does not use any points of $\mathbb{R}^{d}$, other than those in the $n$-tuple argument of $\Theta$, in its definition. Note also that 'affine invariance' of $\Theta$, by which we mean the identity (1) holding for any $\boldsymbol{a}$ in the affine class, is not implied by equivariance. An equivariant transformation $\Theta$ may, however, have the additional property of affine invariance, as in Example 3.

Example 4. An equivariant $\Theta$ illustrating, for $n=2$, a dependence on the order within the $n$-tuple, a feature allowed in [5] but not in [8], is the following 'annulus' centred on $x_{1}$, where $B_{r}(x)$ is the closed ball with centre $x$ and radius $r$ :

$$
\Theta\left(x_{1}, x_{2}\right):=B_{\left\|x_{1}-x_{2}\right\|}\left(x_{1}\right) \backslash B_{\left\|x_{1}-x_{2}\right\| / 2}\left(x_{1}\right) .
$$

There is, of course, no superfluity of arguments.

Example 5. Let $d=2$ and $n=2$ and define $\Delta$ as the rectangle having the two particles as opposite corners and sides parallel to the Cartesian axes. We have equivariance and order invariance; the lack of 'rotational invariance', that is, the failure of (1) to hold when $\boldsymbol{a}$ is an affine transformation with a rotational component, does not invalidate the complementary theorem.

Example 6. Let $\Theta\left(x_{1}, \ldots, x_{n}\right):=\bigcup_{i=1}^{n} B_{1}\left(x_{i}\right)$. This is not equivariant, as the common radius of the balls is fixed in the definition of $\Delta$.

Example 7. Let $d=2$ and $n=2$ and define $\Delta$ as the closed circumdisk having both particles and the origin, $O$, on its boundary. This is not equivariant. Note that a reference point outside the $n$-tuple is used in the construction.

Example 8. Let $d=2$ and $n=3$ and define $\Delta$ as either the circumdisk of the three particles or the domain that we used in Example 1, the choice being decided by a random coin toss. The complementary theorem does not apply here, because $\Theta$ is random.

Example 9. Let $n=3$. Define $\Delta$ by the equivariant map

$$
\Theta\left(x_{1}, x_{2}, x_{3}\right)=B_{\left\|x_{3}-x_{1}\right\| \vee\left\|x_{2}-x_{1}\right\|}\left(x_{1}\right) \backslash B_{\left\|x_{3}-x_{1}\right\| \wedge\left\|x_{2}-x_{1}\right\|}\left(x_{1}\right) .
$$

This is an annulus centred on $x_{1}$ with radii dictated by the other two particles. Thus, the definition is order dependent. We show later that the complementary theorem of earlier authors cannot be applied to this example. 
Example 10. Use the mapping of Example 9 if the volume of the annulus is greater than the volume of its hole; otherwise, define $\Delta:=\varnothing$. This mapping is still equivariant.

Example 11. This is a censored version of Example 3. Define $\Theta$ as in Example 3 when the triangle formed by the 3-tuple is acute angled; if not, define $\Delta:=\varnothing$. The mapping is equivariant and order invariant.

Example 12. Let $n \geq 3$ and $d=2$. Define $\Delta$ as the diametrical disk of $x_{1}$ and $x_{2}$. The remaining points in the $n$-tuple are obviously superfluous; if counted, they lead to a false conclusion regarding the shape parameter of the gamma distribution (except when all $x_{j} \in$ $\Delta, 3 \leq j \leq n)$.

Figure 2 shows, with heavier dots, the germs whose associated domains $\Delta$ (using the map $\Theta$ of Example 4) are 0-filled.

\section{Anchors, marks, and typicality}

The concept of 'typical $n$-tuple' can be best introduced by establishing a marked point process derived from the realised Poisson particle process. We can then draw upon the comprehensive discussion of typicality (within the context of marked point processes) in [11, pp. 105-109], and use notation compatible with that reference.

Let us assign a reference point (or anchor) for each $\Delta$ or, more precisely, for each germ $\left(x_{1}, \ldots, x_{n}\right)$. One choice, used by Miles and named the base particle, is the first component of the $n$-tuple, namely $x_{1}$. More generally, we define an anchor map $z:\left(\mathbb{R}^{d}\right)^{n} \rightarrow \mathbb{R}^{d}$, which is equivariant under scaling and translation; by this we mean (as before) that

$$
z\left(\boldsymbol{a} x_{1}, \boldsymbol{a} x_{2}, \ldots, \boldsymbol{a} x_{n}\right)=\boldsymbol{a} z\left(x_{1}, x_{2}, \ldots, x_{n}\right)
$$

for all transformations $\boldsymbol{a} \in \mathcal{T}$. The base particle is a valid anchor, but sometimes other choices are more natural or more convenient technically. Obviously the mapping $z$ may depend on the order of particles in the germ.

We now focus attention on a new marked point process, in the original space $\mathbb{R}^{d}$, generated from the germs of the product process which have $m$-filled associated domains $\Delta$. Commence by fixing the constants $n$ and $m$ and the mappings $z$ and $\Theta$ in advance. Then, for each germ with an $m$-filled $\Delta$ of positive volume, place a point in $\mathbb{R}^{d}$ at the location of that germ's anchor. Endow that point with a (necessarily positive) mark which is the volume, $V$, of $\Delta$.

For Example 4, in Figure 2 two versions of this marked point process (using $m=0$ ) are shown beneath the product process. The first version uses the base particle as anchor, which is a seemingly natural choice; with this choice, the region in product space where germs have their anchor in $B$ is shown as a lightly shaded rectangle. We also see that the marked point process has points with multiplicities (shown in a vertical stack of dots). Furthermore, each germ contributing to a point's multiplicity provides a mark (shown schematically in Figure 2 by the length of a slanted line extending from the anchor generated by that germ). If we instead define the anchor of $\left(x_{1}, x_{2}\right)$ by $\left(x_{1}+3 x_{2}\right) / 4$, the second version pertains. Note that this has no multiple points and only one, vertically drawn, mark per location.

Why do we emphasise that the mark $V$ must be positive for the inclusion of a marked point at the anchor? This stipulation deals with an issue that arises when $m=0$. Examples 10 and 11 have $\Delta:=\varnothing$ for some $n$-tuples. These empty domains will be 0 -filled with certainty. When $m=0$, our theorems are based (following the style of Miles) on domains of positive volume 
which are 0 -filled by chance, not simply because they are the null set or have zero volume for other reasons (for example being of lower dimension).

Marked point processes were discussed in [11, Section 4.2]. A marked point process is an ordinary point process, $\Psi$ say, on the space $\mathbb{R}^{d} \times \mathbb{M}$, where $\mathbb{M}$ is the mark space (a space endowed with its $\sigma$-algebra $\mathcal{M}$ ). We say that $\mathbb{R}^{d}$ is the carrier space. A marked point process creates a point process in the carrier space, called the projected process, when the marks are ignored.

Our concern is with stationary marked point processes. A marked point process $\Psi$ is stationary if the distribution of $\Psi$ is invariant under any translation applied within the carrier space. In such circumstances, the projected process is also stationary with intensity denoted by (say) $\lambda$. Stationarity implies that, for any $L \in \mathcal{M}, \mathrm{E} \Psi(\cdot, L)$ is proportional to the Lebesgue measure; we let $\lambda_{L}$ be the proportionality constant (so $\lambda$ is short for $\lambda_{\mathbb{M}}$ ).

In general, the projected process may have multiplicities, though this aspect was not emphasised in [11]. The structures in [11] do, however, accommodate multiple points in the projected point process (if the reader thinks of the simple counting-measure space $\mathbb{N}$ in [11] as being allowed multiplicities, as is our $\mathbb{N}$ ). Following [11], let $M$ be the Palm distribution of marks. For each $L \in \mathcal{M}$, this is defined (in the stationary case) as

$$
\begin{aligned}
M(L) & :=\frac{\mathrm{E}(\text { count of points in } B \text { with marks in } L)}{\mathrm{E}(\text { count of points in } B)} \\
& =\frac{\mathrm{E} \Psi(B \times L)}{\mathrm{E} \Psi(B \times \mathbb{M})} \\
& =\frac{\lambda_{L} v_{d}(B)}{\lambda v_{d}(B)} \\
& =\frac{\lambda_{L}}{\lambda}
\end{aligned}
$$

provided that both numerator and denominator are finite and positive. Here $B \in \mathcal{B}^{d}$ is an arbitrary Borel set of positive, finite volume. The typical mark is defined as a random variable on $(\Omega, \mathcal{A}, \mathrm{P})$ having $M(\cdot)$ as its distribution.

We also label our marked point process (marks being positive volumes of $m$-filled domains $\Delta$ placed at $n$-tuple anchors) using the symbol $\Psi$. Now $\mathbb{M}=(0, \infty)$ and a well-behaved Palm distribution (2) satisfying the provision that accompanies (2) gives the following distribution function for marks, for $v>0$ :

$$
\begin{aligned}
F(v): & =\mathrm{P}\{\text { typical mark is less than or equal to } v\} \\
& =M((0, v]) \\
& =\frac{\mathrm{E} \Psi(B \times(0, v])}{\mathrm{E} \Psi(B \times(0, \infty))} \\
& =\frac{\lambda(0, v]}{\lambda}
\end{aligned}
$$

Thus, the typical volume $V$ is defined as a random variable defined on $(\Omega, \mathcal{A}, \mathrm{P})$ and having distribution function given by (3).

Here $\lambda$ is the intensity of anchors for $n$-tuples whose domain $\Delta$ is $m$-filled with positive volume, and $\lambda_{(0, v]}$ is the intensity of the subset whose volume lies in $(0, v]$. Both of these intensities are independent of the choice of $z$ used, a fact proved at the end of this section. Now, 
$\lambda_{(0, v]} v_{d}(B)$ equals

$$
\begin{gathered}
\mathrm{E} \int_{\begin{array}{c}
z\left(x_{1}, \ldots, x_{n}\right) \in B \\
v_{d}\left(\Theta\left(x_{1}, \ldots, x_{n}\right)\right)>0
\end{array}} \mathbf{1}\left(v_{d}\left(\Theta\left(x_{1}, \ldots, x_{n}\right)\right) \leq v,\left(\Phi-\sum_{i=1}^{n} \delta_{x_{i}}\right) \Theta\left(x_{1}, \ldots, x_{n}\right)=m\right) \\
\times \Phi^{[n]}\left(\mathrm{d} x_{1} \times \cdots \times \mathrm{d} x_{n}\right),
\end{gathered}
$$

using the indicator function $\mathbf{1}(\cdot)$. The denominator of (3) can be found similarly, but with the constraint involving $v$ removed. Accordingly,

$$
\begin{aligned}
& \lambda v_{d}(B)=\mathrm{E} \int_{\substack{z\left(x_{1}, \ldots, x_{n}\right) \in B \\
v_{d}\left(\Theta\left(x_{1}, \ldots, x_{n}\right)\right)>0}} \mathbf{1}\left(\left(\Phi-\sum_{i=1}^{n} \delta_{x_{i}}\right) \Theta\left(x_{1}, \ldots, x_{n}\right)=m\right) \\
& \times \Phi^{[n]}\left(\mathrm{d} x_{1} \times \cdots \times \mathrm{d} x_{n}\right) .
\end{aligned}
$$

Our definition (3) for the distribution of volume for $m$-filled domains $\Delta$, augmented by (4) and (5), is essentially the same as that used by Møller and Zuyev [8], except that our technical setting using marked point processes differs from theirs. Moreover, they omitted the conditioning on $\{V>0\}$ and the possible order dependence. There can be problems with definition (3), however, and the similar definitions of [8], if the numerator and denominator in (3) are infinite. We shall see that this is the case in some examples, notably Example 3.

Another representation of the distribution of the 'volume' mark $V$ is given by the moment generating function. Using just $\Delta$ as shorthand for $\Theta\left(x_{1}, \ldots, x_{n}\right)$, without forgetting the dependence on the germ, $\mathrm{Ee}^{s V}$ equals

$$
\frac{1}{\lambda v_{d}(B)} \mathrm{E} \int_{\substack{z\left(x_{1}, \ldots, x_{n}\right) \in B \\ v_{d}(\Delta)>0}} \ldots \int^{s v_{d}(\Delta)} \mathbf{1}\left(\left(\Phi-\sum_{i=1}^{n} \delta_{x_{i}}\right) \Delta=m\right) \Phi^{[n]}\left(\mathrm{d} x_{1} \times \cdots \times \mathrm{d} x_{n}\right) .
$$

Marks can, of course, be more general. The most general for our purposes is the full configuration of each $n$-tuple of particles whose domain $\Delta$ is $m$-filled. The configuration $\boldsymbol{c}_{n}$ of an $n$-tuple lists the position of each particle relative to the anchor $z$; thus, $\boldsymbol{c}_{n}=\left(x_{1}-z, x_{2}-\right.$ $\left.z, \ldots, x_{n}-z\right)$, where order may be important. The space of configurations, denoted by $\mathbb{C}$ with its $\sigma$-algebra $\mathcal{C}$, is the space of all $n$-tuples of points (which may or may not be particles) in $\mathbb{R}^{d}$ whose anchor lies at the origin. Because a configuration is invariant under translations of its defining $n$-tuple in the carrier space, the resulting marked point process, $\Psi^{*}$ say (arising from $n$-tuples of particles whose domains $\Delta$ are $m$-filled with positive volume), is stationary and (2) can be used to provide the distribution, $M^{*}$, of the typical 'configuration mark'. From (2), with $\lambda_{L}$ now equal to $\lambda_{\left\{c_{n}: c_{n} \in L\right\}}$,

$$
M^{*}(L)=\frac{\mathrm{E} \Psi^{*}(B \times L)}{\mathrm{E} \Psi^{*}(B \times \mathbb{M})}=\frac{\lambda_{L}}{\lambda},
$$

where we note that the denominator is the same as in (3). A 'typical configuration' $\boldsymbol{c}_{n}$ is defined as a $\mathbb{C}$-valued random variable on $(\Omega, \mathcal{A}, \mathrm{P})$ having $M^{*}(\cdot)$ as its distribution.

When $V>0$, we may have an interest in $g\left(\boldsymbol{c}_{n}\right)$, where $g$ is a real-valued function on $\mathbb{C} ; V$ itself is an example of such. Note that any function $g$ on $\left(\mathbb{R}^{d}\right)^{n}$ invariant under translations has 
a restriction to $\mathbb{C}$, and that any $g$ on $\mathbb{C}$ has a unique extension to a translation-invariant function on $\left(\mathbb{R}^{d}\right)^{n}$. An expression similar to (6) provides the expectation of $g\left(\boldsymbol{c}_{n}\right)$ (when $\Delta$ is $m$-filled with positive volume, of course):

$$
\begin{gathered}
\mathrm{E} g\left(\boldsymbol{c}_{n}\right)=\frac{1}{\lambda v_{d}(B)} \mathrm{E} \int_{\substack{z\left(x_{1}, \ldots, x_{n}\right) \in B \\
v_{d}(\Delta)>0}} g\left(\boldsymbol{c}_{n}\right) \mathbf{1}\left(\left(\Phi-\sum_{i=1}^{n} \delta_{x_{i}}\right) \Delta=m\right) \\
\times \Phi^{[n]}\left(\mathrm{d} x_{1} \times \cdots \times \mathrm{d} x_{n}\right) .
\end{gathered}
$$

Formula (8) with $g\left(\boldsymbol{c}_{n}\right):=\mathbf{1}\left(\boldsymbol{c}_{n} \in L\right)$ provides a way of calculating (7).

We conclude this section by noting that $\lambda$ and $\lambda_{L}$ (and, hence, $\lambda_{(0, v]}$ ) are independent of the choice of $z$ used. One can prove this using Campbell's theorem for marked point processes (see Equation 4.2.4 of [11]). The equivariant property is an essential condition used in the proof. (Møller [7, p. 46] discussed an equivalent issue for the choice of centroids (i.e. anchors) of the facets in $d$-dimensional tessellations.)

\section{The Mecke-Slivnyak formulae}

The analysis of integrals like those in (4)-(8) would be rather difficult, were it not for an identity established by Mecke [4] (and also establishable from earlier work of Slivnyak [10]). In our context, for a stationary Poisson particle process $\Phi$ of intensity $\rho$ on $\left(\mathbb{R}^{d}, \mathscr{B}^{d}, v_{d}\right)$, Mecke's identity is

$$
\mathrm{E} \int_{\mathbb{R}^{d}} h(x, \Phi) \Phi(\mathrm{d} x)=\rho \int_{\mathbb{R}^{d}} \mathrm{E} h\left(x, \Phi+\delta_{x}\right) v_{d}(\mathrm{~d} x)
$$

for any nonrandom Borel function $h: \mathbb{R}^{d} \times \mathbb{N} \rightarrow[0, \infty)$. The domain of integration stated here is the whole space $\mathbb{R}^{d}$, but could be any nonrandom measurable subset because an indicator function subsumed within the function $h$ would provide the needed restriction. (Mecke also established a converse result: a random counting measure satisfying (9) for all such $h$ is a stationary Poisson process.) Note that, although $h$ is nonrandom in Mecke's identity, $h(x, \Phi)$ inherits the randomness of the counting measure $\Phi$ and is random.

We exploit an extension of this to integrals involving the product process of a stationary Poisson process (an identity used earlier; see [3, p. 53] and [8]), namely

$$
\begin{aligned}
& \mathrm{E} \int_{\left(\mathbb{R}^{d}\right)^{n}} \ldots \int h\left(x_{1}, \ldots, x_{n}, \Phi\right) \Phi^{[n]}\left(\mathrm{d} x_{1} \times \cdots \times \mathrm{d} x_{n}\right) \\
& \quad=\rho^{n} \int_{\left(\mathbb{R}^{d}\right)^{n}} \ldots \int \mathrm{E} h\left(x_{1}, \ldots, x_{n}, \Phi+\sum_{i=1}^{n} \delta_{x_{i}}\right) v_{d}\left(\mathrm{~d} x_{1}\right) \cdots v_{d}\left(\mathrm{~d} x_{n}\right),
\end{aligned}
$$

which holds for any nonrandom Borel function $h:\left(\mathbb{R}^{d}\right)^{n} \times \mathbb{N} \rightarrow[0, \infty)$. This result also applies to nonrandom domains of integration which are subsets of $\left(\mathbb{R}^{d}\right)^{n}$. This important extension is obviously the ideal tool for a study of the complementary theorem, that is, for evaluation of expressions like (4)-(8).

Using (10), with

$$
h\left(x_{1}, \ldots, x_{n}, \Phi\right)=\mathbf{1}\left(z\left(x_{1}, \ldots, x_{n}\right) \in B, v_{d}\left(\Theta\left(x_{1}, \ldots, x_{n}\right)\right)>0, \Phi \Delta=m\right),
$$


from (5) we obtain

$$
\begin{aligned}
\lambda v_{d}(B) & =\rho^{n} \int_{\substack{z\left(x_{1}, \ldots, x_{n}\right) \in B \\
v_{d}\left(\Theta\left(x_{1}, \ldots, x_{n}\right)\right)>0}} \mathrm{E}(\mathbf{1}(\Phi \Delta=m)) v_{d}\left(\mathrm{~d} x_{1}\right) \cdots v_{d}\left(\mathrm{~d} x_{n}\right) \\
& =\rho^{n} \int_{\substack{z\left(x_{1}, \ldots, x_{n}\right) \in B \\
v_{d}\left(\Theta\left(x_{1}, \ldots, x_{n}\right)\right)>0}} \mathrm{P}\{\Phi \Delta=m\} v_{d}\left(\mathrm{~d} x_{1}\right) \cdots v_{d}\left(\mathrm{~d} x_{n}\right) \\
& =\rho^{n} \int_{\substack{z\left(x_{1}, \ldots, x_{n}\right) \in B \\
v_{d}\left(\Theta\left(x_{1}, \ldots, x_{n}\right)\right)>0}} \frac{\left[\rho v_{d}\left(\Theta\left(x_{1}, \ldots, x_{n}\right)\right)\right]^{m}}{m !} \\
& \times \int_{\substack{z\left(u_{1} / \rho^{1 / d}, \ldots, u_{n} / \rho^{1 / d}\right) \in B \\
v_{d}\left(\Theta\left(u_{1} / \rho^{1 / d}, \ldots, u_{n} / \rho^{1 / d}\right)\right)>0}} \frac{\left[\rho v_{d}\left(\Theta\left(u_{1} / \rho^{1 / d}, \ldots, u_{n} / \rho^{1 / d}\right)\right)\right]^{m}}{m !} \\
& \times \exp \left[-\rho v_{d}\left(\Theta\left(\frac{u_{1}}{\rho^{1 / d}}, \ldots, \frac{u_{n}}{\rho^{1 / d}}\right)\right)\right] v_{d}\left(\mathrm{~d} u_{1}\right) \cdots v_{d}\left(\mathrm{~d} u_{n}\right),
\end{aligned}
$$

where we have introduced the change of variable $u_{i}:=\rho^{1 / d} x_{i}$, which implies that $v_{d}\left(\mathrm{~d} u_{i}\right)=$ $\rho v_{d}\left(\mathrm{~d} x_{i}\right)$. Now, using the equivariant properties of the mappings $\Theta$ and $z$, (12) becomes

$$
\begin{aligned}
& \int \ldots \int_{\substack{z\left(u_{1}, \ldots, u_{n}\right) \in \rho^{1 / d} B \\
v_{d}\left(\Theta\left(u_{1}, \ldots, u_{n}\right)\right)>0}} \frac{\left[v_{d}\left(\Theta\left(u_{1}, \ldots, u_{n}\right)\right)\right]^{m}}{m !} \exp \left[-v_{d}\left(\Theta\left(u_{1}, \ldots, u_{n}\right)\right)\right] v_{d}\left(\mathrm{~d} u_{1}\right) \cdots v_{d}\left(\mathrm{~d} u_{n}\right) \\
& \quad=\lambda^{(1)} v_{d}\left(\rho^{1 / d} B\right),
\end{aligned}
$$

where $\lambda^{(\rho)}$ is $\lambda$ augmented by an extra argument to emphasise the intensity of the Poisson process; here, in (13), that intensity is 1 . Thus, (12) and (13) lead to the identity

$$
\lambda^{(\rho)}=\rho \lambda^{(1)} .
$$

Similar change-of-variable arguments and notational augmentations applied to (4) show that

$$
\lambda_{(0, v]}^{(\rho)}=\rho \lambda_{(0, \rho v]}^{(1)},
$$

so we see, from (3), (14), and (15), that

$$
F^{(\rho)}(v)=\frac{\lambda_{(0, v]}^{(\rho)}}{\lambda^{(\rho)}}=\frac{\lambda_{(0, \rho v]}^{(1)}}{\lambda^{(1)}}=F^{(1)}(\rho v) .
$$

The functional relationship (16) does not identify $F^{(\rho)}$, but assists in later theory.

\section{Calculations for some simple examples}

To aid familiarity with our structure, some calculations are desirable. We firstly show the calculations related to Example 4, illustrated in Figure 2. Other examples are discussed; we 
demonstrate that various expressions are infinite in the 'misbehaving' examples. The anchor used in the calculations is always the base particle. The intensity is $\rho$ throughout this section, so the notational augmentation just introduced is not used; absence of the 'intensity superscript' implies that the intensity is $\rho$.

In Example 4, illustrated for $d=1$ in Figure 2, we have $n=2$ and $v_{1}\left(\Theta\left(x_{1}, x_{2}\right)\right)=$ $\left\|x_{1}-x_{2}\right\|$. Choose $z\left(x_{1}, x_{2}\right):=x_{1}$. Then (11) becomes

$$
\begin{aligned}
\lambda \nu_{1}(B) & =\rho^{2} \int_{B} \int_{-\infty}^{\infty} \frac{\left(\rho\left\|x_{1}-x_{2}\right\|\right)^{m}}{m !} \mathrm{e}^{-\rho\left\|x_{1}-x_{2}\right\|} \mathrm{d} x_{2} \mathrm{~d} x_{1} \\
& =2 \rho^{2} \int_{B} \int_{-\infty}^{x_{1}} \frac{\left(\rho\left(x_{1}-x_{2}\right)\right)^{m}}{m !} \mathrm{e}^{-\rho\left(x_{1}-x_{2}\right)} \mathrm{d} x_{2} \mathrm{~d} x_{1} \\
& =2 \rho \int_{B} \int_{0}^{\infty} \frac{\rho^{m+1} u^{m}}{m !} \mathrm{e}^{-\rho u} \mathrm{~d} u \mathrm{~d} x_{1} \\
& =2 \rho v_{1}(B),
\end{aligned}
$$

the integration region in (17) being the lightly shaded region in Figure 2, extending infinitely in the vertical direction (and under the darker region). Thus, $\lambda=2 \rho$. Integration over the intersection of the shaded regions yields a simplified version of (4), where, for $k \geq 1, G_{k, \rho}$ is the distribution function of a $\Gamma(k, \rho)$-distributed variate:

$$
\begin{aligned}
\lambda_{(0, v]} v_{1}(B) & =\rho^{2} \int_{B} \int_{x_{1}-v}^{x_{1}+v} \frac{\left[\rho\left\|x_{1}-x_{2}\right\|\right]^{m}}{m !} \mathrm{e}^{-\rho\left\|x_{1}-x_{2}\right\|} \mathrm{d} x_{2} \mathrm{~d} x_{1} \\
& =2 \rho \int_{B} \int_{0}^{v} \frac{\rho^{m+1} u^{m}}{m !} \mathrm{e}^{-\rho u} \mathrm{~d} u \mathrm{~d} x_{1} \\
& =2 \rho G_{m+1, \rho}(v) v_{1}(B) .
\end{aligned}
$$

Thus, from (3), $F(v)=G_{m+1, \rho}(v)$ and, as anticipated, the distribution of the $m$-filled domain is $\Gamma(m+1, \rho)$.

Example 3 has $d=2$ and $n=3$, and we let $x_{1}$ be the anchor. We have $v_{2}(\Delta)=\frac{1}{2} r_{2} r_{3}|\sin \theta|$, where $r_{i}:=\left\|x_{i}-x_{1}\right\|$ and $\theta$ is the angle $\widehat{x_{3} x_{1} x_{2}}$. So, using these 'polar' coordinates relative to $x_{1}$,

$$
\begin{aligned}
\lambda_{(0, v] \nu_{2}(B)=} \rho^{3} \int_{B} \int_{0}^{\infty}\left(2 \int_{0}^{\pi} \int_{0}^{2 v / r_{2} \sin \theta} \frac{\left(\frac{1}{2} \rho r_{2} r_{3} \sin \theta\right)^{m}}{m !} \mathrm{e}^{-(1 / 2) \rho r_{2} r_{3} \sin \theta} r_{3} \mathrm{~d} r_{3} \mathrm{~d} \theta\right) \\
\times 2 \pi r_{2} \mathrm{~d} r_{2} \nu_{2}\left(\mathrm{~d} x_{1}\right) \\
=2 \rho^{3} \int_{B} \int_{0}^{\infty} \frac{4(m+1) G_{m+2, \rho}(v)}{r_{2}^{2} \rho^{2}}\left(\int_{0}^{\pi} \frac{\mathrm{d} \theta}{\sin ^{2} \theta}\right) 2 \pi r_{2} \mathrm{~d} r_{2} v_{2}\left(\mathrm{~d} x_{1}\right) .
\end{aligned}
$$

We note that the innermost integral is divergent, so the numerator of (3) is infinite, as is the denominator (shown by (18) with $v$ replaced by $\infty$ ). The integral diverges because of the contribution from a vast number of very long, thin triangles that are $m$-filled and of small area. The substantial effect of these very elongated triangles has been overlooked by others studying the problem; in effect, huge numbers of particles $x_{2}$ and $x_{3}$ (at least one of which may be a large distance from $x_{1}$ ) must be counted. 
The analysis for Example 2 proceeds in the same way, but with

$$
\nu_{2}(\Delta)=\frac{\pi}{4 \sin ^{2} \theta}\left(r_{2}^{2}+r_{3}^{2}-2 r_{2} r_{3} \cos \theta\right) .
$$

The calculations become lengthy and are thus omitted, but the large area of $\Delta$ when $x_{2}$ and/or $x_{3}$ are distant from $z$, combined with the $\mathrm{e}^{-\rho \text { area( }(\Delta)}$ weighting of the integrand, mitigates against the influence of distant particles $x_{2}$ and $x_{3}$ on the results. Similar considerations apply in Example 1. Both the numerator and denominator of (3) are finite in these two examples.

In Example 9, $n=3$ and we set $d=2$ for simplicity. Now $v_{2}(\Delta)=\pi\left|r_{2}^{2}-r_{3}^{2}\right|$, where $r_{i}:=\left\|x_{i}-x_{1}\right\|$, so

$$
\begin{aligned}
& \lambda_{(0, v]} \nu_{2}(B) \\
& =\rho^{3} \int_{B} 2 \int_{0}^{\infty}\left(\int_{r_{2}}^{\sqrt{r_{2}^{2}+v / \pi}} \frac{\left(\rho \pi\left(r_{3}^{2}-r_{2}^{2}\right)\right)^{m}}{m !} \mathrm{e}^{-\rho \pi\left(r_{3}^{2}-r_{2}^{2}\right)} 2 \pi r_{3} \mathrm{~d} r_{3}\right) 2 \pi r_{2} \mathrm{~d} r_{2} v_{2}\left(\mathrm{~d} x_{1}\right) \\
& =\rho^{2} \int_{B} 2 G_{m+1, \rho}(v) \int_{0}^{\infty} 2 \pi r_{2} \mathrm{~d} r_{2} v_{2}\left(\mathrm{~d} x_{1}\right) .
\end{aligned}
$$

This is infinite, as is the equivalent result when $v \rightarrow \infty$. The reader will be aware, from elementary considerations, that such an annulus (the domain $\Delta)$ has a $\Gamma(m+1, \rho)$-distributed area, when $m$-filled. The complementary theorem would suggest a $\Gamma(m+2, \rho)$ distribution if it were carelessly invoked. Also note that if $\Delta$ were redefined to be the 'hole' in the annulus, of area $\pi\left(r_{1} \wedge r_{2}\right)^{2}$, then the theorem would still not apply, as the numerator and denominator of (3) would still be infinite.

Example 10 introduces a constraint on Example 9 which prevents domains being very thin annuli of very large radii - the abundance of these being the reason for the pathology of Example 9. Indeed, $\Delta=\varnothing$ unless the area, $\pi\left[\left(r_{3} \vee r_{2}\right)^{2}-\left(r_{3} \wedge r_{2}\right)^{2}\right]$, of the annulus is greater than the area, $\pi\left(r_{3} \wedge r_{2}\right)^{2}$, of its hole. Thus, and here we note that the integrating condition $\left\{v_{d}(\Delta)>0\right\}$ affects the result for the first time,

$$
\begin{aligned}
\lambda_{(0, v]} & =2 \rho^{3} \int_{0}^{\sqrt{v / \pi}}\left(\int_{\sqrt{2} r_{2}}^{\sqrt{r_{2}^{2}+v / \pi}} \frac{\left(\rho \pi\left(r_{3}^{2}-r_{2}^{2}\right)\right)^{m}}{m !} \mathrm{e}^{-\rho \pi\left(r_{3}^{2}-r_{2}^{2}\right)} 2 \pi r_{3} \mathrm{~d} r_{3}\right) 2 \pi r_{2} \mathrm{~d} r_{2} \\
& =2 \rho^{2} \int_{0}^{\sqrt{v / \pi}}\left(G_{m+1, \rho}(v)-G_{m+1, \rho}\left(\pi r_{2}^{2}\right)\right) 2 \pi r_{2} \mathrm{~d} r_{2} \\
& =2 \rho^{2}\left(v G_{m+1, \rho}(v)-\int_{0}^{v} G_{m+1, \rho}(u) \mathrm{d} u\right) \\
& =2 \rho^{2} \int_{0}^{v} u d G_{m+1, \rho}(u) \\
& =2(m+1) \rho G_{m+2, \rho}(v) \\
& \rightarrow 2(m+1) \rho, \quad v \rightarrow \infty,
\end{aligned}
$$

where we have removed the easily evaluated outer integral with respect to $x_{1}$. We see that the constraint has brought the example back into line with the complementary theorem: $F(v)=$ $G_{m+2, \rho}(v)$ and $\lambda=2(m+1) \rho$.

Finally we consider the important Example 11, which has been discussed extensively by the other authors. We might anticipate that the taboo on triangles with an obtuse angle 
would eliminate the problems seen in Example 3. This turns out to be the case. We impose on Example 3 the 'acute angles conditions' $\{\theta<\pi / 2\} \cup\{\theta>3 \pi / 2\},\left\{r_{3}>r_{2} \cos \theta\right\}$, and $\left\{r_{2}>r_{3} \cos \theta\right\}$. Applying these conditions yields

$$
\begin{aligned}
\lambda & =\rho^{3} \int_{0}^{\infty}\left(2 \int_{0}^{\pi / 2} \int_{r_{2} \cos \theta}^{r_{2} / \cos \theta} \frac{\left(\frac{1}{2} \rho r_{2} r_{3} \sin \theta\right)^{m}}{m !} \mathrm{e}^{-(1 / 2) \rho r_{2} r_{3} \sin \theta} r_{3} \mathrm{~d} r_{3} \mathrm{~d} \theta\right) 2 \pi r_{2} \mathrm{~d} r_{2} \\
& =4 \pi \rho \int_{0}^{\infty}\left(\int_{0}^{\pi / 2} \frac{4(m+1)\left[G_{m+2, \rho}\left(\frac{1}{2} r_{2}^{2} \tan \theta\right)-G_{m+2, \rho}\left(\frac{1}{2} r_{2}^{2} \cos \theta \sin \theta\right)\right]}{r_{2} \sin ^{2} \theta} \mathrm{d} \theta\right) \mathrm{d} r_{2} \\
& =16 \pi(m+1) \rho \int_{0}^{\pi / 2}\left(\int_{0}^{\infty} \frac{\left[G_{m+2, \rho}\left(\frac{1}{2} r_{2}^{2} \tan \theta\right)-G_{m+2, \rho}\left(\frac{1}{2} r_{2}^{2} \cos \theta \sin \theta\right)\right]}{r_{2} \sin ^{2} \theta} \mathrm{d} r_{2}\right) \mathrm{d} \theta \\
& =16 \pi(m+1) \rho \int_{0}^{\pi / 2} \frac{\log (\sec \theta)}{\sin ^{2} \theta} \mathrm{d} \theta \\
& =8 \pi^{2}(m+1) \rho \\
& <\infty .
\end{aligned}
$$

Thus, this 'censored' example now lies within the domain of the existing complementary theorem. The abundance of very thin, very long triangles which created the pathology of Example 3 has been removed, so the other authors have been correct in quoting results based on the complementary theorem. Incidentally, the amazing simplification of the integral with respect to $r_{2}$, leading to a result independent of $m$ and $\rho$ even though the integrand depends on both, was found with the help of MATHEMATICA ${ }^{\circledR}$; space does not permit a proof.

\section{The change-of-measure technique yields a general proof}

Up to this point, the Poisson process $\Phi$ has had intensity $\rho$, except in certain comparative statements ((14)-(16)). This can be cemented notationally by indexing the probability measure $\mathrm{P}$ by $\rho$. Thus, each $\mathrm{P}$ and $\mathrm{E}$ in the earlier sections (except those used in (14)-(16), where intensity changes can be viewed in a different way (see below)) can be read as $\mathrm{P}_{\rho}$ and $\mathrm{E}_{\rho}$. From (8), we obtain

$$
\begin{aligned}
& \mathrm{E}_{\rho} g\left(\boldsymbol{c}_{n}\right) \\
& =\frac{1}{\lambda^{(\rho)} v_{d}(B)} \mathrm{E}_{\rho} \int_{\substack{z\left(x_{1}, \ldots, x_{n}\right) \in B \\
v_{d}(\Delta)>0}} g\left(\boldsymbol{c}_{n}\right) \mathbf{1}\left(\left(\Phi-\sum_{i=1}^{n} \delta_{x_{i}}\right) \Delta=m\right) \Phi^{[n]}\left(\mathrm{d} x_{1} \times \cdots \times \mathrm{d} x_{n}\right) \\
& =\frac{\rho^{n}}{\lambda^{(\rho)} v_{d}(B)} \int_{z\left(x_{1}, \ldots, x_{n}\right) \in B} \ldots \int_{v_{d}(\Delta)>0} g\left(\boldsymbol{c}_{n}\right) \frac{\left[\rho v_{d}(\Delta)\right]^{m}}{m !} \mathrm{e}^{-\rho v_{d}(\Delta)} v_{d}\left(\mathrm{~d} x_{1}\right) \cdots v_{d}\left(\mathrm{~d} x_{n}\right) \\
& =\frac{\rho^{n+m} \lambda^{(\tau)}}{\tau^{n+m} \lambda^{(\rho)}} \times \frac{\tau^{n}}{\lambda(\tau)} v_{d}(B) \\
& \quad \times \int_{z\left(x_{1}, \ldots, x_{n}\right) \in B} \ldots \int_{v_{d}(\Delta)>0} g\left(\boldsymbol{c}_{n}\right) \mathrm{e}^{(\tau-\rho) v_{d}(\Delta)} \frac{\left[\tau v_{d}(\Delta)\right]^{m}}{m !} \mathrm{e}^{-\tau v_{d}(\Delta)} v_{d}\left(\mathrm{~d} x_{1}\right) \cdots v_{d}\left(\mathrm{~d} x_{n}\right) \\
& =\left(\frac{\rho}{\tau}\right)^{n+m-1} \mathrm{E}_{\tau} g\left(\boldsymbol{c}_{n}\right) \mathrm{e}^{(\tau-\rho) V} .
\end{aligned}
$$


In particular, following Møller and Zuyev [8], who derived (20) within their slightly more restrictive setting, we consider the Laplace transform of $V$ using $\mathrm{E}_{1}$, that is,

$$
\mathrm{E}_{1} \mathrm{e}^{-s V}=\mathrm{E}_{1} \mathrm{e}^{(1-(1+s)) V}=\frac{1}{(1+s)^{n+m-1}} \mathrm{E}_{1+s} 1=\frac{1}{(1+s)^{n+m-1}} .
$$

Therefore, $F^{(1)}(v)=G_{n+m-1,1}(v)$. Invoking the functional relationship (16) yields $F^{(\rho)}(v)=$ $G_{n+m-1, \rho}(v)$ and, hence, the traditional complementary theorem stated in Theorem 1.

In closing this section, we note that the interplay between intensities $\rho$ and $\tau$ can be discussed in different ways. In this section, we have two probability measures, $\mathrm{P}_{\rho}$ and $\mathrm{P}_{\tau}$, and one pointprocess mapping, $\Phi$. In Section 4 , the reader may have envisaged one probability measure $\mathrm{P}$ and two point process mappings with different intensities, $\rho$ and 1. Either approach is valid and the results of each section can be written in the language of the other.

\section{Independence of volume $V$ and configuration $c_{n}$}

Both Miles [5] and Møller and Zuyev [8] noted a type of independence between the volume, $V$, and the configuration, $\boldsymbol{c}_{n}$, of a typical $m$-filled $n$-tuple. Recall that $\boldsymbol{c}_{n}:=\left(x_{1}-z, x_{2}-z\right.$, $\left.\ldots, x_{n}-z\right)$. In this section, we clarify the nature of this independence and when it occurs, within our context. There are also new independence results, one based on the notion of an 'equivariant subset' of the configuration space $\mathbb{C}$.

Lemma 1. For a typical $n$-tuple configuration $\boldsymbol{c}_{n}$ whose associated equivariant domain $\Delta$ has positive volume $V$ and is $m$-filled, the random variate $V$ and any other real-valued random variable $X$ are independent if and only if the distribution of $X$ does not depend on $\rho$.

Proof. Using (20), we obtain

$$
\mathrm{E}_{1} \mathrm{e}^{-s V-t X}=\mathrm{E}_{1} \mathrm{e}^{(1-(1+s)) V} \mathrm{e}^{-t X}=\frac{1}{(1+s)^{n+m-1}} \mathrm{E}_{1+s} \mathrm{e}^{-t X}=\mathrm{E}_{1}\left(\mathrm{e}^{-s V}\right) \mathrm{E}_{1+s}\left(\mathrm{e}^{-t X}\right) .
$$

The result follows from this identity.

Theorem 2. In the context of Lemma $1, V$ is independent of

- $\mathbf{1}\left(\boldsymbol{c}_{n} / V^{1 / d} \in L\right)$, for any $L \in \mathcal{C}$ (as stated in [8, Theorem 4]);

- $\mathbf{1}\left(\boldsymbol{c}_{n} / W^{1 / d} \in L\right)$, for any $L \in \mathcal{C}$, where $W$ is the volume of the smallest d-dimensional ball which has each of $x_{1}, x_{2}, \ldots, x_{\min (d+1, n)}$ on its boundary (as implicitly stated in [5, Theorem 5.1]);

- $\mathbf{1}\left(\rho^{1 / d} \boldsymbol{c}_{n} \in L\right)$, for any $L \in \mathcal{C}$;

- $\mathbf{1}\left(\boldsymbol{c}_{n} \in L\right)$, for any equivariant $L \in \mathcal{C}$, where $L$ is called equivariant if and only if $\boldsymbol{c}_{n} \in L$ implies that $\boldsymbol{a c}_{n} \in L$ for any transformation $\boldsymbol{a} \in \mathcal{T}$ and any configuration $\boldsymbol{c}_{n}$.

Remark 1. The first two statements establish results of the cited works within our context. The last two statements are new. 
Proof of Theorem 2. From (8) and (10), we obtain

$$
\begin{aligned}
& \lambda^{(\rho)} v_{d}(B) \mathrm{E}_{\rho}\left(\mathbf{1}\left(\frac{\boldsymbol{c}_{n}}{V^{1 / d}} \in L\right)\right) \\
& =\rho^{n} \int_{\substack{z\left(x_{1}, \ldots, x_{n}\right) \in B \\
v_{d}\left(\Theta\left(x_{1}, \ldots, x_{n}\right)>0\right.}} \mathbf{1}\left(\frac{\boldsymbol{c}_{n}}{V^{1 / d}} \in L\right) \mathrm{E}_{\rho}(\mathbf{1}(\Phi \Delta=m)) v_{d}\left(\mathrm{~d} x_{1}\right) \cdots v_{d}\left(\mathrm{~d} x_{n}\right) \\
& =\rho^{n} \int_{\substack{z\left(x_{1}, \ldots, x_{n}\right) \in B \\
\nu_{d}\left(\Theta\left(x_{1}, \ldots, x_{n}\right)>0\right.}} \mathbf{1}\left(\frac{\left(x_{1}-z\left(x_{1}, \ldots, x_{n}\right), \ldots, x_{n}-z\left(x_{1}, \ldots, x_{n}\right)\right)}{V\left(x_{1}, \ldots, x_{n}\right)^{1 / d}} \in L\right) \\
& \times \frac{\left[\rho v_{d}\left(\Theta\left(x_{1}, \ldots, x_{n}\right)\right)\right]^{m}}{m !} \exp \left[-\rho v_{d}\left(\Theta\left(x_{1}, \ldots, x_{n}\right)\right)\right] v_{d}\left(\mathrm{~d} x_{1}\right) \cdots v_{d}\left(\mathrm{~d} x_{n}\right) \\
& =\rho^{n} \quad \int_{z\left(u_{1} / \rho^{1 / d}, \ldots, u_{n} / \rho^{1 / d}\right) \in B} \\
& v_{d}\left(\Theta\left(u_{1} / \rho^{1 / d}, \ldots, u_{n} / \rho^{1 / d}\right)>0\right. \\
& \times \mathbf{1}\left(\left[\left(\frac{u_{1}}{\rho^{1 / d}}-z\left(\frac{u_{1}}{\rho^{1 / d}}, \ldots, \frac{u_{n}}{\rho^{1 / d}}\right), \ldots, \frac{u_{n}}{\rho^{1 / d}}-z\left(\frac{u_{1}}{\rho^{1 / d}}, \ldots, \frac{u_{n}}{\rho^{1 / d}}\right)\right)\right]\right. \\
& \left.\times\left[V\left(\frac{u_{1}}{\rho^{1 / d}}, \ldots, \frac{u_{n}}{\rho^{1 / d}}\right)^{1 / d}\right]^{-1} \in L\right) \\
& \times \frac{\left[\rho v_{d}\left(\Theta\left(u_{1} / \rho^{1 / d}, \ldots, u_{n} / \rho^{1 / d}\right)\right)\right]^{m}}{m !} \\
& \times \exp \left[-\rho v_{d}\left(\Theta\left(\frac{u_{1}}{\rho^{1 / d}}, \ldots, \frac{u_{n}}{\rho^{1 / d}}\right)\right)\right] v_{d}\left(\mathrm{~d} u_{1}\right) \cdots v_{d}\left(\mathrm{~d} u_{n}\right) \\
& =\int \ldots \int_{\substack{z\left(u_{1}, \ldots, u_{n}\right) \in \rho^{1 / d} B \\
v_{d}\left(\Theta\left(u_{1}, \ldots, u_{n}\right)>0\right.}} \mathbf{1}\left(\frac{\left(u_{1}-z\left(u_{1}, \ldots, u_{n}\right), \ldots, u_{n}-z\left(u_{1}, \ldots, u_{n}\right)\right)}{V\left(u_{1}, \ldots, u_{n}\right)^{1 / d}} \in L\right) \\
& \times \frac{\left[v_{d}\left(\Theta\left(u_{1}, \ldots, u_{n}\right)\right)\right]^{m}}{m !} \exp \left[-v_{d}\left(\Theta\left(u_{1}, \ldots, u_{n}\right)\right)\right] v_{d}\left(\mathrm{~d} u_{1}\right) \cdots v_{d}\left(\mathrm{~d} u_{n}\right) \\
& =\lambda^{(1)} v_{d}\left(\rho^{1 / d} B\right) \mathrm{E}_{1}\left(\mathbf{1}\left(\frac{\boldsymbol{c}_{n}}{V^{1 / d}} \in L\right)\right) \text {. }
\end{aligned}
$$

In view of (14), $\mathbf{1}\left(\boldsymbol{c}_{n} / V^{1 / d} \in L\right)$ satisfies the requirements of the variate $X$ in Lemma 1 . The first assertion of the theorem is thus proved. The remaining assertions are proved in a similar way.

Intuitively, a constraint $L$ on the configuration is equivariant if it constrains only the shape of the configuration. For example, $L:=\left\{\boldsymbol{c}_{3}\right.$ : the three points form an acute-angled triangle $\}$ is an equivariant constraint.

\section{Miles' method has similar problems}

If a marked point process $\Psi$ is ergodic - and all of our processes are (due to the mixing character of Poisson processes) - then there is a definition of the typical mark alternative to that given via (2). 
Without loss of generality, we can take the reference domain $B$ to be the ball $B_{R} \equiv B_{R}(O)$ of radius $R$ and centre $O$. When $R$ is large, the number, $\Psi(B \times \mathbb{M})$, of points in $B$ is large, with a fairly representative collection of marks. The proportion, $\Psi(B \times L) / \Psi(B \times \mathbb{M})$, of these whose marks lie within $L \in \mathcal{M}$ approximates the mark distribution $M(L)$. As $R \rightarrow \infty$, the approximation converges almost surely to $M(L)$ (see [11, p. 108]), i.e.

$$
M(L):=\lim _{R \rightarrow \infty}\left(\frac{\Psi\left(B_{R} \times L\right)}{\Psi\left(B_{R} \times \mathbb{M}\right)}\right) .
$$

It is assumed that, when $R$ is finite, the numerator and denominator in (22) are finite with probability 1 .

The theory of Miles [5] is based on this ergodic definition of the typical configuration of $n$ particles (which have an $m$-filled associated domain $\Delta$ ). Miles' anchor is always the base particle.

His theory has difficulties, however, in situations like those of Examples 2 and 8 . In Example 2, for example, the number of $m$-filled triangles with base particle in $B_{R}$ is infinite with probability 1 , for any $R$.

\section{A definition of typicality when $\lambda$ is infinite}

In the pathological cases where existing definitions do not apply, we typically find both infinite numerator and denominator in (3). This suggests the following approach.

Instead of the marked point process $\Psi$, we use an alternative marked point process $\Psi_{R}$ (or $\Psi_{R}^{(\rho)}$ if $\rho$ requires emphasis). We construct this as follows: for each $n$-tuple whose domain $\Delta$ has positive volume $V$ and is $m$-filled, we place a point (with mark V) at the $n$-tuple's anchor $z$ if and only if all particles of the $n$-tuple are in the ball $B_{R}(z)$.

The typical mark has a distribution given, from the arguments leading to (3), by

$$
F_{R}^{(\rho)}(v):=\frac{\mathrm{E} \Psi_{R}^{(\rho)}(B \times(0, v])}{\lambda^{(\rho, R)} v_{d}(B)}=\frac{\lambda_{(0, v]}^{(\rho, R)}}{\lambda^{(\rho, R)}}, \quad v>0 .
$$

The expressions on the right-hand side of (23) are the obvious extensions of earlier notation and it is easy to adapt arguments (12)-(16) to show the following comparative relationships:

$$
\begin{gathered}
\lambda^{(\rho, R)}=\rho \lambda^{\left(1, \rho^{1 / d} R\right)}, \\
\lambda_{(0, v]}^{(\rho, R)}=\rho \lambda_{(0, \rho v]}^{\left(1, \rho^{1 / d} R\right)}, \\
F_{R}^{(\rho)}(v)=F_{\rho^{1 / d} R}^{(1)}(\rho v) .
\end{gathered}
$$

The change-of-measure result, (20), also takes a revised form in this new context:

$$
\begin{aligned}
\mathrm{E}_{\rho}^{(R)} g\left(\boldsymbol{c}_{n}\right) & =\left(\frac{\rho}{\tau}\right)^{n+m} \frac{\lambda^{(\tau, R)}}{\lambda^{(\rho, R)}} \mathrm{E}_{\tau}^{(R)} g\left(\boldsymbol{c}_{n}\right) \mathrm{e}^{(\tau-\rho) V} \\
& =\left(\frac{\rho}{\tau}\right)^{n+m-1} \frac{\lambda^{\left(1, \tau^{1 / d} R\right)}}{\lambda^{\left(1, \rho^{1 / d} R\right)}} \mathrm{E}_{\tau}^{(R)} g\left(\boldsymbol{c}_{n}\right) \mathrm{e}^{(\tau-\rho) V} .
\end{aligned}
$$


Furthermore, (21) becomes

$$
\begin{aligned}
\mathrm{E}_{1}^{(R)} \mathrm{e}^{-s V} & =\mathrm{E}_{1}^{(R)}\left(\mathrm{e}^{(1-(1+s)) V}\right) \\
& =\frac{1}{(1+s)^{n+m-1}} \frac{\lambda^{\left(1,(1+s)^{1 / d} R\right)}}{\lambda^{(1, R)}} \mathrm{E}_{1+s}^{(R)} 1 \\
& =\frac{1}{(1+s)^{n+m-1}} \frac{\lambda^{\left(1,(1+s)^{1 / d} R\right)}}{\lambda^{(1, R)}} .
\end{aligned}
$$

The introduction of the 'bounding' ball $B_{R}(z)$ suggests the following new definition of typicality for the marks of the 'unbounded' process $\Psi$. The distribution function $F$ for the typical mark in $\Psi$ is now defined by

$$
F(v):=\lim _{R \rightarrow \infty} F_{R}(v), \quad v>0,
$$

whenever this limit exists. This agrees with (3) when $\lambda$ is finite and extends the definition otherwise. In view of (24), the distribution function $F$ will clearly depend on how the moderating factor,

$$
\lim _{R \rightarrow \infty} \frac{\lambda^{\left(1,(1+s)^{1 / d} R\right)}}{\lambda^{(1, R)}},
$$

behaves.

In making this definition, the author is aware of some limitations. To date, we have not established in any complete fashion that this definition is consistent with other similar definitions where, for example, the 'bounding domains' are not balls but instead form some other nest of increasing domains which eventually fill the space. Nor have we established that (26) is independent of the choice of $z$ in all cases. Further work is needed, but since the recognition that the classical complementary theorem is not universally valid is an important finding, we shall proceed to use the new definition given in (25). In Section 12, we discuss this issue further.

\section{Application to the examples}

Examples using this new definition will help cement ideas. In the mundane Example 4, we can readily calculate that $\lambda^{(1, R)}$ equals $2 G_{m+1,1}(R)$. Therefore, the moderating factor for this example is 1 . In the 'misbehaving' Example 9 with $d=2$, following the logic of (19) we obtain

$$
\begin{aligned}
\lambda^{(1, R)} & =2 \int_{0}^{R}\left(\int_{r_{2}}^{R} \frac{\left(\pi\left(r_{3}^{2}-r_{2}^{2}\right)\right)^{m}}{m !} \mathrm{e}^{-\pi\left(r_{3}^{2}-r_{2}^{2}\right)} 2 \pi r_{3} \mathrm{~d} r_{3}\right) 2 \pi r_{2} \mathrm{~d} r_{2} \\
& =2 \int_{0}^{R} G_{m+1,1}\left(\pi\left(R^{2}-r_{2}^{2}\right)\right) 2 \pi r_{2} \mathrm{~d} r_{2} \\
& =2 \int_{0}^{\pi R^{2}} G_{m+1,1}(u) \mathrm{d} u \\
& =2\left[\pi R^{2} G_{m+1,1}\left(\pi R^{2}\right)-(m+1) G_{m+2,1}\left(\pi R^{2}\right)\right] .
\end{aligned}
$$

Recalling that $d=2$, the moderating factor can be calculated as follows:

$$
\begin{aligned}
\frac{\lambda^{\left(1,(1+s)^{1 / d} R\right)}}{\lambda^{(1, R)}} & =\frac{2\left[\pi(1+s) R^{2} G_{m+1,1}\left(\pi(1+s) R^{2}\right)-(m+1) G_{m+2,1}\left(\pi(1+s) R^{2}\right)\right]}{2\left[\pi R^{2} G_{m+1,1}\left(\pi R^{2}\right)-(m+1) G_{m+2,1}\left(\pi R^{2}\right)\right]} \\
& \rightarrow(1+s), \quad R \rightarrow \infty .
\end{aligned}
$$


Thus, from (24), we have $\mathrm{E}_{1} \mathrm{e}^{s V}=1 /(1+s)^{n+m-2}$ and, therefore, substituting $n=3$, $F^{(1)}(v)=G_{m+1,1}(v)$ as claimed in Section 5. Thus, the volume of an $m$-filled annulus is $\Gamma(m+1, \rho)$-distributed.

In the interesting Example 3, (18) is modified as follows:

$$
\begin{aligned}
\lambda^{(1, R)} & =\int_{0}^{R}\left(2 \int_{0}^{\pi} \int_{0}^{R} \frac{\left(\frac{1}{2} r_{2} r_{3} \sin \theta\right)^{m}}{m !} \mathrm{e}^{-(1 / 2) r_{2} r_{3} \sin \theta} r_{3} \mathrm{~d} r_{3} \mathrm{~d} \theta\right) 2 \pi r_{2} \mathrm{~d} r_{2} \\
& =16 \pi(m+1) \int_{0}^{\pi}\left(\int_{0}^{R} \frac{G_{m+2,1}\left(\frac{1}{2} r_{2} R \sin \theta\right)}{r_{2}} \mathrm{~d} r_{2}\right) \frac{\mathrm{d} \theta}{\sin ^{2} \theta} \\
& =32 \pi(m+1) \int_{0}^{\pi / 2}\left(\int_{0}^{(1 / 2) R^{2} \sin \theta} \frac{G_{m+2,1}(u)}{u} \mathrm{~d} u\right) \frac{\mathrm{d} \theta}{\sin ^{2} \theta} \\
& =32 \pi(m+1) \int_{0}^{(1 / 2) R^{2}} \frac{G_{m+2,1}(u)}{u}\left(\int_{\sin ^{-1}\left(2 u / R^{2}\right)}^{\pi / 2} \frac{\mathrm{d} \theta}{\sin ^{2} \theta}\right) \mathrm{d} u \\
& =16 \pi(m+1) R^{2} \int_{0}^{(1 / 2) R^{2}} \frac{G_{m+2,1}(u)}{u^{2}} \sqrt{1-\frac{4 u^{2}}{R^{4}}} \mathrm{~d} u .
\end{aligned}
$$

We shall show, using the dominated convergence theorem, that

$$
\lim _{R \rightarrow \infty} \int_{0}^{\infty} \mathbf{1}\left(\left[0, \alpha R^{2}\right]\right) \frac{G_{m+2,1}(u)}{u^{2}} \sqrt{1-\frac{u^{2}}{\alpha^{2} R^{4}}} \mathrm{~d} u=\int_{0}^{\infty} \frac{G_{m+2,1}(u)}{u^{2}} \mathrm{~d} u=\frac{1}{m+1}
$$

for any $\alpha>0$. An integrable function, $f$ say, which dominates the integrand for all $R$ is

$$
f(u):= \begin{cases}\frac{u^{m}}{(m+2) !}, & 0 \leq u \leq 1, \\ \frac{1}{u^{2}}, & u>1 .\end{cases}
$$

This is so because

$$
\mathbf{1}\left(\left[0, \alpha R^{2}\right]\right) \frac{G_{m+2,1}(u)}{u^{2}} \sqrt{1-\frac{u^{2}}{\alpha^{2} R^{4}}}<\frac{G_{m+2,1}(u)}{u^{2}}<\frac{1}{u^{2}}
$$

(the final inequality holding for $u>1$ ), since $G_{m+2,1}$ is a distribution function, and

$$
\frac{G_{m+2,1}(u)}{u^{2}}=\frac{1}{u^{2}} \int_{0}^{u} \frac{t^{m+1}}{(m+1) !} \mathrm{e}^{-t} \mathrm{~d} t<\frac{1}{u^{2}} \int_{0}^{u} \frac{t^{m+1}}{(m+1) !} \mathrm{d} t=\frac{u^{m}}{(m+2) !} .
$$

By substituting $d=2$ and $\alpha=\frac{1}{2}$ into (27), we obtain the denominator of the moderating factor. The numerator is obtained with $\alpha=(1+s)^{2 / d}$. Therefore, the moderating factor in (26) is $1+s$. Thus, $\mathrm{E}_{1} \mathrm{e}^{s V}=1 /(1+s)^{n+m-2}$ and, therefore, $F^{(1)}(v)=G_{m+1,1}(v)$ as claimed in Section 1.

The moderating factor can be $(1+s)^{\kappa}$, for $\kappa>1$, as in the following three examples.

Example 13. For general $n \geq 2$ and $d$, let $\Delta$ be the closed ball $B_{\min _{i>1}\left(r_{i}\right)}\left(x_{1}\right)$, where $r_{i}:=$ $\left\|x_{i}-x_{1}\right\|$. Here $\kappa=n-2$ and, so, can be large.

Example 14. For $n=4$ and $d=2$, let $\Delta$ be the triangle with vertices $x_{1}, x_{2}$, and $x_{3}$ if $x_{4}$ is not contained therein. Otherwise, $\Delta$ is the triangle with vertices $x_{1}, x_{2}$, and $x_{4}$. Then we can show that $\kappa=2$. 
Example 15. For $n=4$ and $d=2$, let $\Delta$ be the triangle of smallest area using three of the four available particles. We conjecture that $\kappa=2$ in this case.

Details of the proofs of these statements are omitted. Their provision is left as an exercise for the reader.

\section{A revised complementary theorem}

We can summarise our theory and arguments, based on the definition of typicality given in Section 9, as follows.

Theorem 3. (Revised complementary theorem.) When the intensity $\lambda$ is infinite in the context of Theorem 1, the Laplace transform of $V$ is given by

$$
\frac{1}{(1+s)^{n+m-1}} \lim _{R \rightarrow \infty} \frac{\lambda^{\left(1,(1+s)^{1 / d} R\right)}}{\lambda^{(1, R)}},
$$

involving the so-called moderating factor defined in (26).

One might hope that the moderating factor always takes the form $(1+s)^{\kappa}$, for $\kappa \geq 0$, but the author has been unable, to date, to prove this for general choices of $\Theta, d, n$, and $m$. If the moderating factor does always take this form, then the volume of $V$ retains its gamma distribution.

\section{Discussion: open questions}

Clearly Theorem 3 is uncontroversial in circumstances where $\lambda$ is finite, for then it reduces to the classical theorem (Theorem 1). In that case, there is no issue concerning the choice of anchor $z$, used as the centre of our nest of bounding balls.

In the other cases, we should perhaps consider different choices of $z$. In Example 3, the distributional results concerning the area of $\Delta$ remain unchanged when the analysis is repeated with $z$ defined as the circumcentre of the three particles, using a parametrisation based on Santaló [9, Equation 2.18].

It is noteworthy, however, that questions pertaining to the configuration shape may depend on $z$, and this can be of great importance in examples where $\Theta$ is defined as $\varnothing$ for some configurations. This must be done, as in Example 11, in such a way as to conserve the equivariant property of the mapping. This means that the constraint $L:=\left\{\boldsymbol{c}_{n}: \Delta=\varnothing\right\}$ must be equivariant.

A question then arises: What is the probability that the typical $n$-tuple has an associated domain $\Delta$ which is not $\varnothing$ ? In Example 11, this question changes: What is the probability that a typical 3-tuple of particles from a Poisson process on the plane forms a triangle which is acute angled? Miles [5] and Santaló [9, pp. 16-17] stated that this probability is $\frac{1}{2}$. Note that the question answered by these authors is not cast in terms of $m$-filled triangles; all triangles are under consideration, regardless of their filling.

We approach this problem within the confines of a marked point process constructed as follows. A point is placed in $\mathbb{R}^{2}$ at the anchor $z:=x_{1}$ of the 3-tuple if and only if all three particles lie in the closed ball $B_{R}(z)$. The mark placed at this location is the configuration $c_{n}$. Note that this marked point process is the superposition of a countable collection of marked point processes, the $m$ th based on the $m$-filled condition. Thus, $\lambda^{(1, R)}$ is the sum of the respective 
left-hand sides of (27) for $m \geq 0$, whence

$$
\lambda^{(1, R)}=8 \pi R^{2} \int_{0}^{(1 / 2) R^{2}} \sqrt{1-\frac{4 u^{2}}{R^{4}}} \mathrm{~d} u=\pi^{2} R^{4},
$$

an obvious result. The probability that the typical mark lies in $L:=\left\{c_{3}: \Delta\right.$ is acute angled $\}$ is $\lambda_{L}^{(1, R)} / \lambda^{(1, R)}$, where

$$
\begin{aligned}
\lambda_{L}^{(1, R)} & =\int_{0}^{R} 2 \int_{0}^{\pi / 2} \int_{r_{2} \cos \theta}^{\min \left(R, r_{2} / \cos \theta\right)} r_{3} \mathrm{~d} r_{3} \mathrm{~d} \theta 2 \pi r_{2} \mathrm{~d} r_{2} \\
& =2 \pi \int_{0}^{\pi / 2}\left(\int_{0}^{R \cos \theta}\left(\frac{r_{2}^{2}}{\cos ^{2} \theta}-r_{2}^{2} \cos ^{2} \theta\right) r_{2} \mathrm{~d} r_{2}+\int_{R \cos \theta}^{R}\left(R^{2}-r_{2}^{2} \cos ^{2} \theta\right) r_{2} \mathrm{~d} r_{2}\right) \mathrm{d} \theta \\
& =\frac{1}{4} \pi^{2} R^{4} .
\end{aligned}
$$

Thus, for all $R>0$, the probability that the 3-tuple forms an acute-angled triangle is $\frac{1}{4}$. If we define the probability that the typical 3-tuple satisfies $L$ as being the limit of $\lambda_{L}^{(1, R)} / \lambda^{(1, R)}$ as $R \rightarrow \infty$, then we obtain the result $\frac{1}{4}$.

We get an entirely different answer, however, if $z$ is defined to be the circumcentre of the three particles: the answer is now $\frac{1}{3}$. It is of considerable interest that this shape entity depends on the choice of $z$ while the distribution of the area of $\Delta$ does not. In Section 7, where we set out the independence of shape and size, we explained this intuitively.

Close inspection of the statements of Miles and Santaló reveal a reason for their answer being $\frac{1}{2}$ : they have actually answered a different question. Santaló's calculation [9, pp. 1617], which, incidentally, has a number of errors that cancel each other and, so, do not distort his final answer, is restricted to 3-tuples whose circumdisk lies wholly within $B_{R}$, with the circumcentre anywhere within $B_{R}$. This removes from consideration many obtuse triangles, creating a huge bias in favour of acute-angled triangles. Miles focused his attention on 3-tuples whose circumradius is less than a constant, $R_{0}$; this creates a similar bias.

Our results of $\frac{1}{4}$ and $\frac{1}{3}$ also differ from the probability that three points uniformly and independently distributed within a ball form an acute-angled triangle. Hall [2] showed this to be $\xi=4 / \pi^{2}-\frac{1}{8}=0.2803$. So shape issues are delicate, size issues less so.

In conclusion, further work is needed on Theorem 3, but the results of this paper correct certain misapprehensions which have appeared in the literature. With these corrected, progress in the right direction is possible.

\section{Acknowledgements}

I thank the two referees for their helpful comments on an earlier draft.

\section{References}

[1] Cowan, R., Quine, M. And Zuyev, S. (2003). Decomposition of Gamma-distributed domains constructed from Poisson point processes. Adv. Appl. Prob. 35, 56-69.

[2] Hall, G. R. (1982). Acute triangles in the $n$-ball. J. Appl. Prob. 19, 712-715.

[3] Kallenberg, O. (1976). Random Measures. Springer, Berlin.

[4] Mecke, J. (1967). Stationäre zufällige Maße auf lokalkompakten Abelschen Gruppen. Z. Wahrscheinlichkeitsth. 9, 36-58.

[5] Miles, R. E. (1970). On the homogeneous planar Poisson process. Math. Biosci. 6, 85-127.

[6] Miles, R. E. (1971). Poisson flats in Euclidean spaces. II. Homogeneous Poisson flats and the complementary theorem. Adv. Appl. Prob. 3, 1-43. 
[7] Møller, J. (1989). Random tessellations in $\mathbb{R}^{d}$. Adv. Appl. Prob. 21, 37-73.

[8] Møller, J. And Zuyev, S. (1996). Gamma-type results and other related properties of Poisson processes. $A d v$. Appl. Prob. 28, 662-673.

[9] Santaló, L. A. (1976). Integral Geometry and Geometric Probability (Encyclopaedia Math. Appl. 1). AddisonWesley, Reading, MA.

[10] Slivnyak, I. M. (1962). Some properties of stationary flows of homogeneous random events. Theory Prob. Appl. 7, 336-341.

[11] Stoyan, D., Kendall, W. S. And Mecke, J. (1995). Stochastic Geometry and Its Applications, 2nd edn. John Wiley, Chichester.

[12] Zuyev, S. (1999). Stopping sets: gamma-type results and hitting properties. Adv. Appl. Prob. 31, 355-366. 